\title{
Idiopathic Sialectasia of Stensen's Duct Treated by Marsupialisation of Ectatic Segment
}

\section{Indranil Pal, ${ }^{1}$ Saumitra Kumar, ${ }^{1}$ Anindita Sinhababu, ${ }^{2}$ Kushal Chatterjee ${ }^{3}$}

\section{Introduction}

\section{ABSTRACT}

Sialectasis of parotid duct is an uncommon condition characterised by abnormal dilatation of a part or whole of the Stensen's duct. It may lead to recurrent parotid infections as well as a cause of concern for the patient. Superficial parotidectomy is the accepted surgical treatment for this condition.

\section{Case Report}

A case of idiopathic dilatation of parotid duct is presented. It was treated by marsupialisation of the sialectatic segment.This procedure proved to be much simpler and safer than superficial parotidectomy whilst being equally effective.

\section{Conclusion}

In cases of Stensen's duct sialectasis without any abnormality of the parotid gland, marsupialisation of the ectatic segment should be the treatment of choice.

Keywords:

Parotid Gland; Salivary Ducts; Dilatation, pathologic

$\mathrm{T}$ The term sialectasis refers to dilation of the salivary ducts. Sialectasis of the Stensen's duct is an infrequent condition, characterized by the dilatation of a segment of a salivary duct. The dilatation can result from intra ductal papillomas, ${ }^{1}$ sialoliths or kinks and partial strictures of the duct. ${ }^{2}$ Ductal stenosis may occur secondary to sialolithotomy, traumatic ductal injury with resultant fibrosis, or as a consequence of long standing ductal inflammation associated with chronic parotitis3. We describe a case of parotid duct sialectasis where the cause of the dilatation is not clear. It was managed surgically with good results. The case is reported for its rarity and uncertain etiology. A review of

1 - Department of ENT, College of Medicine and JNM Hospital, Kalyani, West Bengal

2 - Department of Pathology, College of Medicine and JNM Hospital, Kalyani, West Bengal

3 - Department of Dentistry, College of Medicine and JNM Hospital, Kalyani, West Bengal

\section{Corresponding author:}

Dr Saumitra Kumar

email: saumitra80@gmail.com literature revealed only 5 articles depicting its occurrence in humans. ${ }^{3}$

\section{Case Report}

A 25 years old man presented at the Otorhinolaryngology outpatient clinic with a gradually increasing painless swelling on his left cheek in front of his left ear for the preceding 10 days. He reported repeated similar episodes over the past one year which culminated with discharge of watery material from the swelling into his mouth on massaging the swelling. The size of the swelling was unrelated to mastication. There was no history of pain, trauma or surgical intervention in the cheek or parotid region.

On examination there was a $2 \mathrm{~cm} \times 2 \mathrm{~cm}$ soft, non tender and fluctuant swelling on the left cheek which was bimanually palpable, though not evident on visual inspection. The intra oral parotid duct opening was normal in appearance. The overlying buccal mucosa and skin of the cheek were normal in appearance. Intraoral 
digital pressure on the swelling produced a watery discharge from the parotid duct opening with collapse of the swelling.

The Stensen's duct was cannulated with a $24 \mathrm{G}$ intravenous cannula and a sialography performed. No resistance was encountered while inserting the cannula. The sialogram (Fig. 1) revealed a dilatation of the Stensen's duct some distance proximal to its opening into the oral cavity.

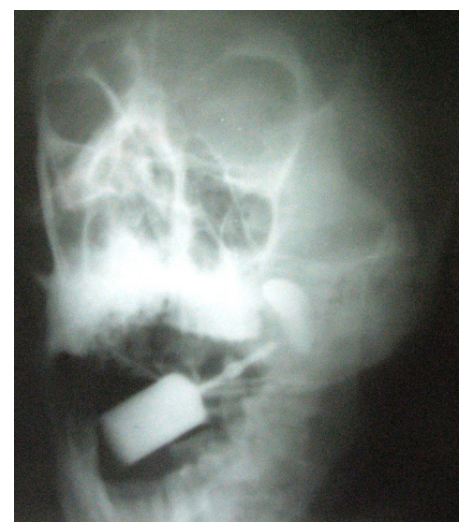

Fig. 1 Sialogram showing fusiform dilatation of the Stensen's duct

After local anaesthesia with infiltration of $2 \%$ lignocaine and 1/100,000 adrenaline solution, the parotid (Stensen's) duct was cannulated with a 24 $\mathrm{G}$ intravenous cannula and trypan blue solution was injected into the duct. A vertical semilunar incision, with an anterior convexity was made on the buccal mucosa about $1 \mathrm{~cm}$ in front of the parotid duct opening (Fig.2). The Stensen's duct opening was retracted medially with a 3-0 silk stay suture and using a small hemostat the dilated duct was dissected out. A vertical incision was made on the lateral wall of the dilated duct and the ductal ectasia opened up. The posterior lip of the incised duct was sutured to the anterior lip of the buccal mucosal incision. The Stensen's duct opening along with a large portion of the medial wall of the ectatic segment and the overlying mucosa was excised and the margins of the ectatic duct mucosa sutured with the surrounding buccal mucosa. The ecstatic duct was thus marsupialised (Fig.3). The surgical site was dressed with a bolster sutured over it. The bolster was removed after 24 hours. Post operative recovery was uneventful. The sutured

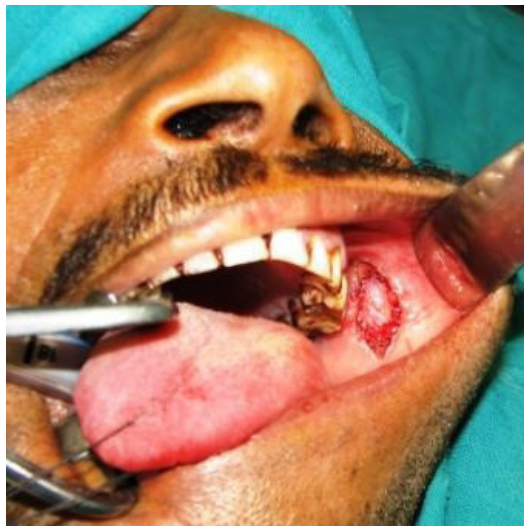

Fig. 2 Semilunar incision made anterior to Stensen's duct opening

lining of the ectatic segment became continuous with the buccal mucosa and the proximal end of the ectatic segment became the new parotid duct opening into the buccal mucosa.

The patient had no recurrence of the condition till 2 years of follow up.

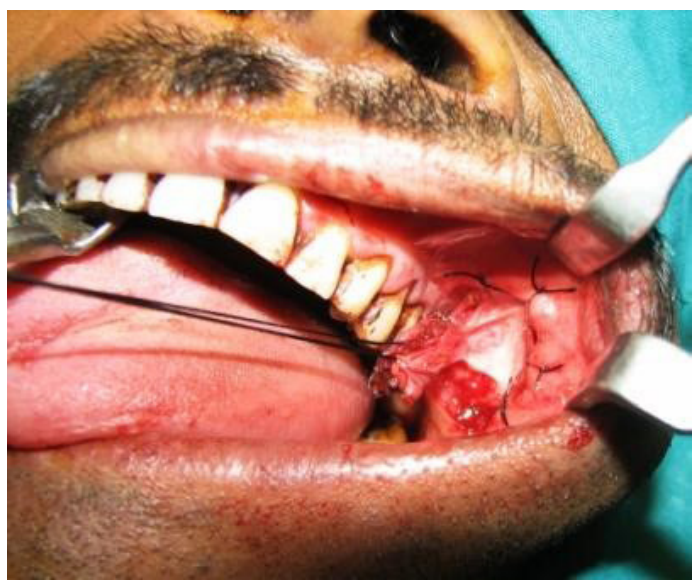

Fig. 3 Marsupialisation of ectatic segment

Histopathological examination of the excised segment after processing and staining with hematoxylin and eosin (H\&E) showed a cystically dilated duct, mostly lined by flattened and cuboidal epithelium with focal areas of columnar epithelium (Fig. 4). The segment between the dilatation and buccal opening of the Stensen's duct was carefully examined after serial sections but no apparent cause of the dilatation of the duct (Obstructive lesion) 


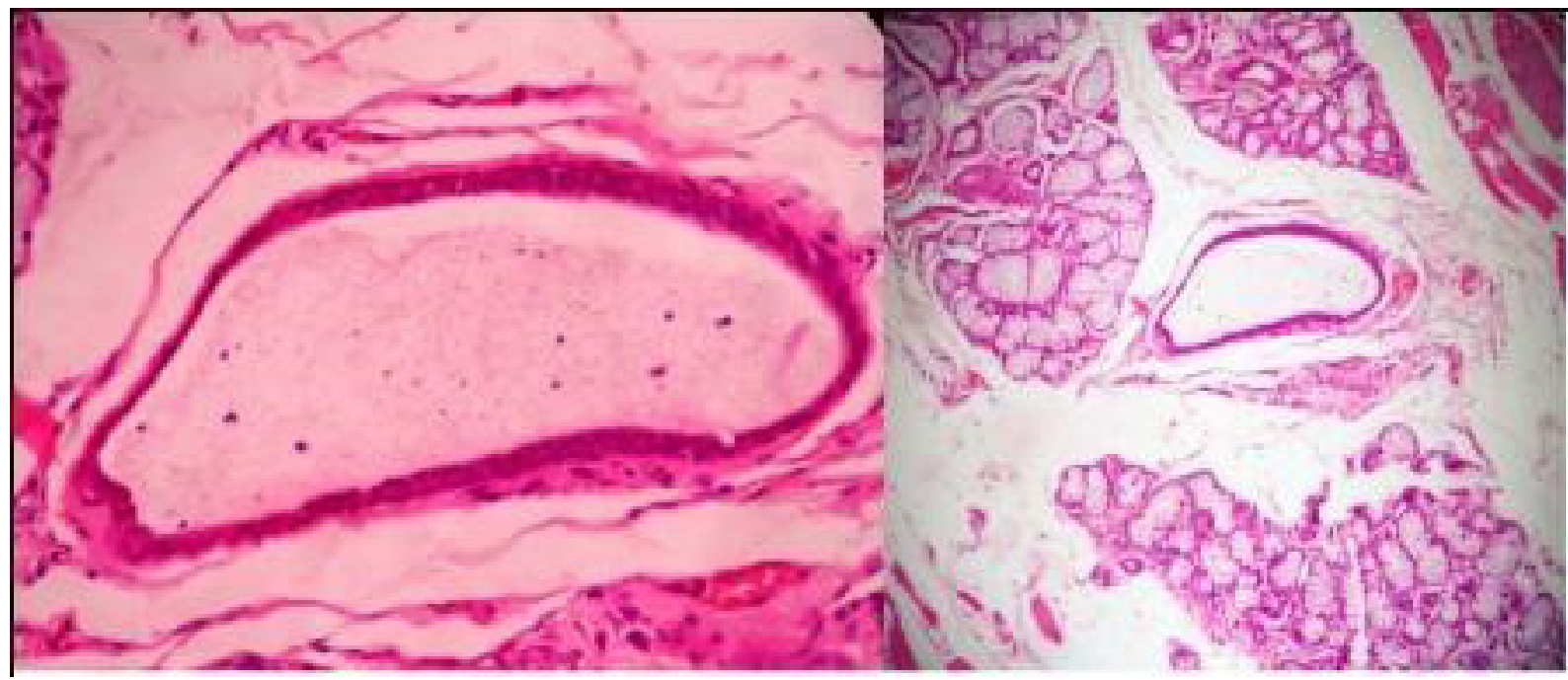

Fig. 4 (L)--Dilated duct lined by flattened to cuboidal epithelium with focal areas of columnar epithelium (400X, H\&E). (R)--A cystically dilated duct amidst normal lobules of acini, (100X, H\&E)

could be identified.

\section{Discussion}

The term "sialectasis" synonymous with "ptyalectasis" denotes an abnormal dilatation of a salivary duct. It presents itself as recurrent painful swellings during mastication and/or swallowing. Parotid duct ectasia is a fairly rare condition characterized by the dilatation of a segment of the parotid duct usually secondary to some kind of obstructive pathology in the duct downstream. The obstruction can be due to intra ductal papillomas, ${ }^{4}$ sialoliths or kinks and partial strictures of the duct. ${ }^{2}$ Ductal stenosis may also occur secondary to sialolithotomy, traumatic ductal injury or bite injury to the Stensen's duct opening with resultant fibrosis, or as a consequence of long standing ductal inflammation associated with chronic parotitis. ${ }^{3}$ The majority of the dilatations of salivary ducts in the major salivary glands are within the glands and the dilatation of the Stensen's duct is very rare. In the case presented here the ductal dilatation began about $1 \mathrm{~cm}$ proximal to the Stensen's duct opening in the buccal mucosa. We didn't find any intra luminal obstruction in the distal undilated segment. Neither did histopathological examination show any obstructive lesion, fibrosis or otherwise on the walls of the duct or outside. The lining of the dilated segment also didn't show any abnormality indicating any kind of weakness of the duct wall. There was also no history of any pain on the affected side of the face or neck ever, as far as the patient could recall.

There is another condition called Congenital dilatation of Stensen's duct (CDSD) which is a rare heteroplasia of the parotid gland, which may have a hereditary background. ${ }^{5,6}$ It presents as a painless and progressive swelling in the cheek along the Stensen's duct without an obvious cause. The static secretions undergo secondary infection causing pain, fever, and intraoral purulent flow from Stensen's duct. Combining the literature with their experience, Wang et al (2011) summarized the diagnostic features of CDSD as follows: 1) the primary symptom of painless swelling in the cheek that is not related to eating, without any evident etiology; 2) may be unilateral or bilateral, and may occur in any age group; 3 ) clinically, the presence of swelling along the Stensen's duct (in patients without a history of inflammation, aggressive massage of the swelling can produce abundant intraoral salivary flow); and 4) parotid sialography demonstrates dilated Stensen's duct with a smooth margin but no evidence of obstruction. Our patient fits the above mentioned diagnostic criteria for CDSD. However, CDSD patients show a tube-like mobile swelling in the cheek area following the entire route of Stensen's duct. Our patient 
showed an immobile, eccentric saccular dilatation of only a segment of the Stensen's duct. The dilation can affect the main duct or the terminal duct. Our patient showed an immobile, eccentric saccular dilatation of only a segment of the Stensen's duct. Though Wang et al recommended that CDSD patients be treated with superficial parotidectomy, we found the dilated segment easily accessible intraorally and so performed a more conservative marsupialisation of the dilated segment with satisfactory results. ${ }^{?}$

We presume that there was some kind of partial obstruction of the affected salivary duct probably by a calculus which led to the duct ectasia. The obstruction must have thereafter cleared spontaneously to leave behind the permanently dilated segment without any symptoms of pain. The dilatation was responsible for the stasis of salivary secretions and since the patient could massage out the static salivary secretions, there was no microbial colonization of the fluid leading to infection and pain. Sialoendoscopy directly visualises the ductal system for any intraductal pathology. But sialoendoscopes are currently unavailable in our institution. Since we are unable to find a proper cause for the dilatation, our diagnosis can be termed as idiopathic dilatation of the Stensens duct. ${ }^{8}$ Other differential diagnoses included pneumatocele, sialocele, lipoma, dental infection, juvenile recurrent parotitis (JRP) and chronic obstructive parotitis. JRP is the second most frequently encountered inflammatory disease of salivary glands in children next to mumps. JRP usually starts before adolescence and like in our case demonstrates sialectases in terminal ducts. But, it is characterized by repeated (usually unilateral) parotid swellings associated with fever, pain, and malaise. The condition is self-limiting after adolescence in more than $90 \%$ of cases. Pneumatocele is seen in glass blowers and musicians who blow wind instruments. The patient had a clear history of painless fluid collection which could be expressed by application of digital pressure. Moreover, he did not have any history of blowing or antecedent trauma.This rules out JRP, lipoma, dental infection as well as pneumatocele. A sialocele is a cystic cavity containing saliva. It usually results from trauma or infection to the parotid gland parenchyma, laceration of the parotid duct or ductal stenosis with subsequent dilatation. Our patient denied any history of trauma and the collection could be expressed through the Stensen's duct opening ruling out a sialocele. Moreover the histopathology of the excised segment of his Stensen's duct showed normal features and no evidence of any obstruction or duct wall weakness. We came to the conclusion that he had an idiopathic dilatation of the Stensen's duct.

Several treatment options are available for this condition. Conservative methods like repeated aspiration, compression, balloon dilatation under fluoroscopic guidance with stenting, sialoendoscopy with saline under pressure do not have very predictable results. Excision of the dilated portion of the duct with stent placement and suturing of the ductal orifice to the buccal mucosa or open ductal exploration and parotidectomy are more aggressive procedures. $^{3}$ Aggressive surgical treatment is reserved for traumatic sialectasis. ${ }^{9,10}$ Two reports show excellent results from marsupialisation of the dilated segment of the duct. ${ }^{3,8}$

Though rare this entity needs to be diagnosed timely to prevent recurrent parotitis and treated accordingly. We also depict that a simple operative procedure like marsupialisation should be preferred over superficial parotidectomy in uncomplicated cases. It is easier, safer and operative time is significantly lesser.

\section{References}

1. Alho OP, Kristo A, Luotonen J, et al. Intraductal papilloma as a cause of a parotid duct cyst: A case report. J Laryngol Otol. 1996; 110:277

2. Laudenbach P, Fain J, Canet E. Duct cysts of the parotid Complications of dilated Stensen's duct. Rev Stomatol ChirMaxillofac. 1990; 91:78

3. Baurmash HD. Sialectasis of Stensen's duct with an extraoral swelling: a case report with surgical management. J Oral Maxillofac Surg. 2007 Jan;65(1):140-3

4. Alho OP, Kristo A, Luotonen J, et al. Intraductal papilloma as a cause of a parotid duct cyst: A case report. J Laryngol Otol. 1996;110:277

5. Yu GY. Diseases of the Salivary Glands. Beijing, China. Union Publisher of Peking Medical University and Peking Union Medical College; 1994:58-59.

6. Wang SL. Non-Neoplastic Diseases of the Salivary Glands. 
Beijing, China. Scientific and Technical Documents Publishing House; 2001:82-83

7. Wang Y, Yu GY, Huang MX, Mao C, Zhang L. Diagnosis and treatment of congenital dilatation of Stensen's duct. Laryngoscope 2011;121(8):1682-6. doi: 10.1002/lary.21854..

8. Shivangi Lohia, Arjun S Joshi. Idiopathic sialectasis of the Stensen's duct treated with marsupialisation. BMJ Case Reports
2013; doi:10.1136/bcr-2013-201548

9. Lewkowicz AA, Hasson O, Nahlieli O. Traumatic injuries to the parotid gland and duct. J Oral Maxillofac Surg. 2002; 60:676-80.

10. Canosa A, Cohen MA. Post-traumatic parotid sialocele: report of two cases. J Oral Maxillofac Surg. 1999; 57:742-5. 